\title{
ASSESSMENT OF THE MOMENT OF STABILITY WHEN PROVIDING FRONTAL STABILITY OF THE EXOSKELETON
}

\author{
Yuri Andrianov*, Pyotr Fishchenko, Alexander Kapustin \\ Volga State University of Technology, Russian Federation
}

In medical exoskeletons, it is necessary to support a stable vertical position of a person. Based on the use of mathematical modeling and theoretical mechanics methods, estimation of the moment of stability is performed while ensuring the frontal (lateral) stability of a person in a medical exoskeleton. This will allow to receive the maximum allowable safe speed, eliminating the fall in the frontal plane. Recommendations for providing frontal (lateral) stability are given.

Key words: medical exoskeleton, frontal stability of the exoskeleton, the moment of stability of the exoskeleton, the balance of the exoskeleton, supporting the balance of the exoskeleton

\section{INTRODUCTION}

Medical exoskeletons for legs are intended for rehabilitation of patients with disorders of the musculoskeletal system. The exoskeleton is an open kinematic chain with six degrees of freedom, three degrees of freedom for each pedipulator. With the help of special fastenings, the exoskeleton is fixed on the waist and the legs of a person. The medical exoskeleton models the movements that are identical to the locomotor movements of a person: straight walking, sitting and resting on a support, walking up the stairs, walking along a curve, etc. The need to maintain the vertical stability of the human-exoskeleton system (exosystem) is a feature of such exoskeletons. In exoskeletons of supporting or strengthening type, vertical stability is provided by a person with a healthy vestibular apparatus. Vice versa, in medical exosystems the exoskeleton imitates human movements. This means that the structure of the medical exoskeleton must include some device that provides vertical stability.

Considering the mechanical system of a person in an exoskeleton, we have a system of two masses, a person and an exoskeleton connected to each other by an elastic linkage. Maintaining the vertical stability of such a system is a complex non-trivial task [1]. The general model of the exosystem can be considered an inverted pendulum with one and two degrees of freedom and is well described in the works of A.M. Formal'sky [2]. However, practical implementation of the 'vestibular' apparatus of the exoskeleton is very complicated.

Existing ways to maintain vertical stability can be divided into:

- passive - with the help of additional supporting elements and not related to the adjustment of the exoskeleton limbs movement: crutches, suspension system, parapod;

- active, where sustainability can be provided by electric drives of the exoskeleton or introduction of additional degrees of freedom [3, 4];

- structural, where stability can be provided by a large mass of the exoskeleton, a significant footprint surface area, or massive moving elements correcting the total center of gravity.

The proposed system of maintaining vertical stability refers to structural ones and presents a rotary support device with the possibility of shifting the center of gravity of the payload against the axis of the transport platform through kinematically connected to each other drive beveled rings with the possibility of their relative rotation [Patent RU 159557 U1].

The aim of the work was to estimate approximately the change in the magnitude of the moment of stability while ensuring the frontal (lateral) stability of the exoskeleton in case of using rotary support.

\section{THEORY AND METHODS}

The exosystem should have a stationary property of the system [5], i.e. lateral stability allowing a person with an exoskeleton neither to fall down nor to fall on its side when moving. Ensuring lateral stability can be written as

$m \cdot \frac{v^{2}}{R} \cdot H \leq P \cdot \frac{L}{2}$

where $m$ is the exosystem mass, $v$ is the velocity of its center of mass, $\mathrm{R}$ is the radius of curvature of the trajectory, $P$ is the weight of the exosystem, $L$ is the width of the base platform, $\mathrm{H}$ is the height of the inertia force above the horizontal support area creating a tilting moment, Fig. 1.

The tilting moment tends to tilt and turn over the exoskeleton on its side around a straight line running along the edge of the base platform. Usually this straight line is parallel to the horizontal longitudinal axis of the moving base platform and to the speed.

The right side of the inequation determines the magnitude of the moment of stability.

One of the ways to ensure the stability of the exosystem can be a shift in the center of gravity and a change in the moment of stability tending to prevent a fall in the 


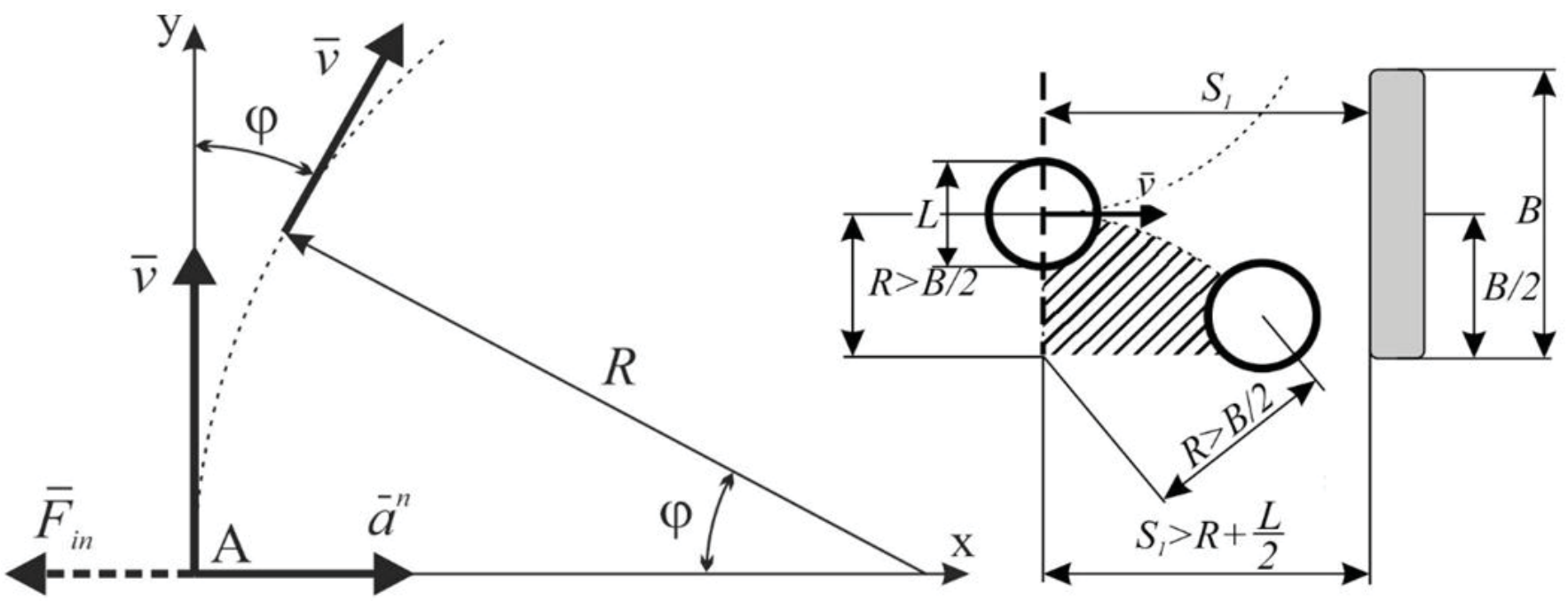

Figure1: Options for ensuring lateral stability

frontal plane. Structurally, the displacement of the center of gravity is provided by means of a rotating device with a wedge ring.

The tilting moment tends to tilt and turn over the exoskeleton on its side around a straight line running along the edge of the base platform. Usually this straight line is parallel to the horizontal longitudinal axis of the moving base platform and to the speed.

The right side of the inequation determines the magnitude of the moment of stability.

One of the ways to ensure the stability of the exosystem can be a shift in the center of gravity and a change in the moment of stability tending to prevent a fall in the frontal plane. Structurally, the displacement of the center of gravity is provided by means of a rotating device with a wedge ring.

\section{EXPERIMENT}

A prototype of a medical exoskeleton was developed in the design bureau of the university for experimental studies. A description of the exoskeleton Remotion (Fig. 2) and its work can be found in $[6,7]$. The manufacture of the medical exoskeleton was carried out with the imple-
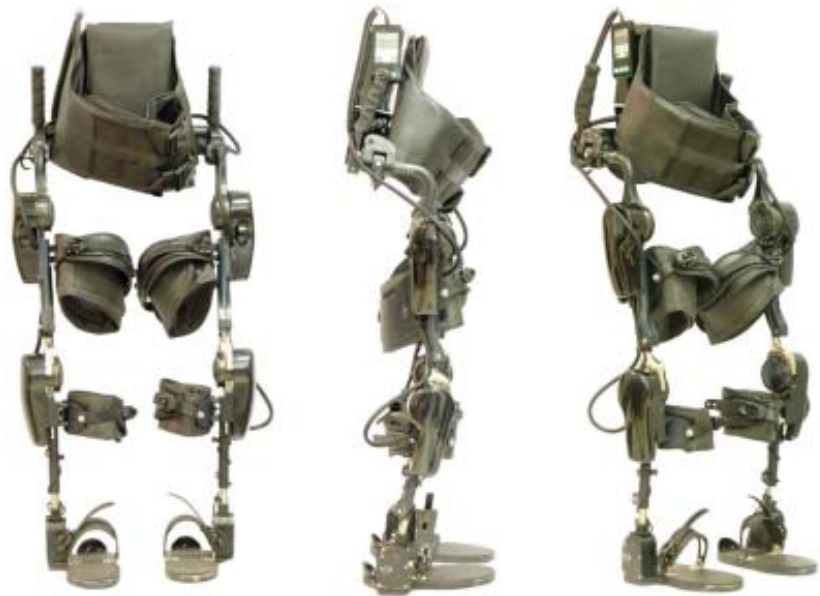

Figure 2: General view of Remotion exoskeleton mentation of a complex project on creation of high-tech production with the support of the Government of the Russian Federation.

In the designed device, the payload - the control unit and the power supply unit - are placed together on the rotary support. When turning the upper part of the rotator, the center of gravity shifts, which allows to increase the magnitude of the moment of stability.

\section{RESULTS AND DISCUSSION}

Fig. 3 shows the initial and final positions (cross section of the YOZ plane) of the rotary support after the upper part $B_{2}^{\prime} K N E_{2}^{\prime}$ is rotated by $180^{\circ}$ around $\mathrm{OZ}_{1}$ axis, constituting $y$ angle with vertical axis $O Z\left(O Z_{1}\right.$ axis together with normal vector $\bar{N}$ is perpendicular to plane $A B_{2} D E_{2}$ ). The center of gravity of the load moves from initial position $C_{W}$ to position $C{ }_{W}$ which is symmetrical to the initial

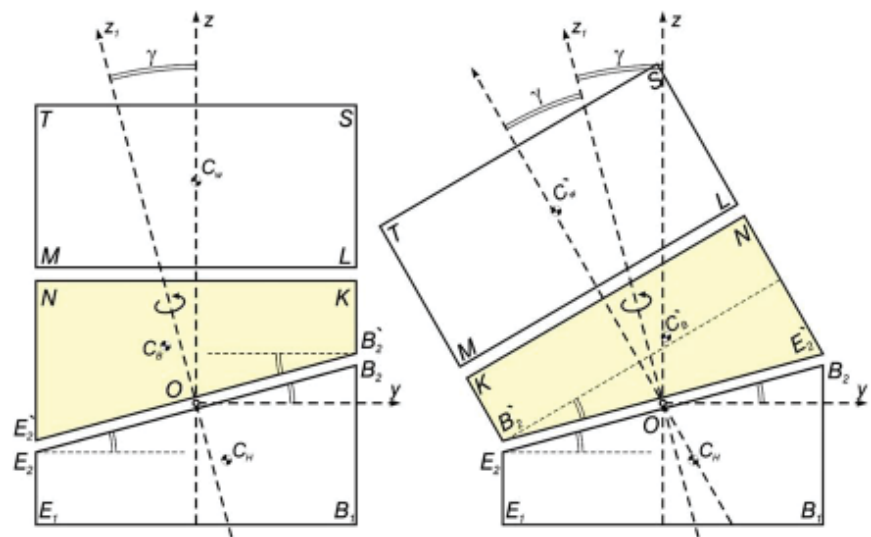

Figure 3: Inclination of TSLM load at an angle of $2 \gamma$ when turning by $180^{\circ}$

one relative to axis $O Z_{1}$. The result is a change in the shoulder of the moment of stability.

Fig. 4 shows a diagram of the lower part of the rotary support and the position of the centers of gravity of three conventional parts (they are supposed to be made of one homogeneous material). 
The coordinates of the center of gravity $C_{1}$ of cylinder $A B D E A_{1} B_{1} D_{1} E_{1}$ and its volume equal respectively to

$x_{1}=0 ; y_{1}=0 ; z_{1}=-h_{1} / 2$;

$V_{1}=\pi R^{2} h_{1}$, where $h_{1}=B B_{1}$

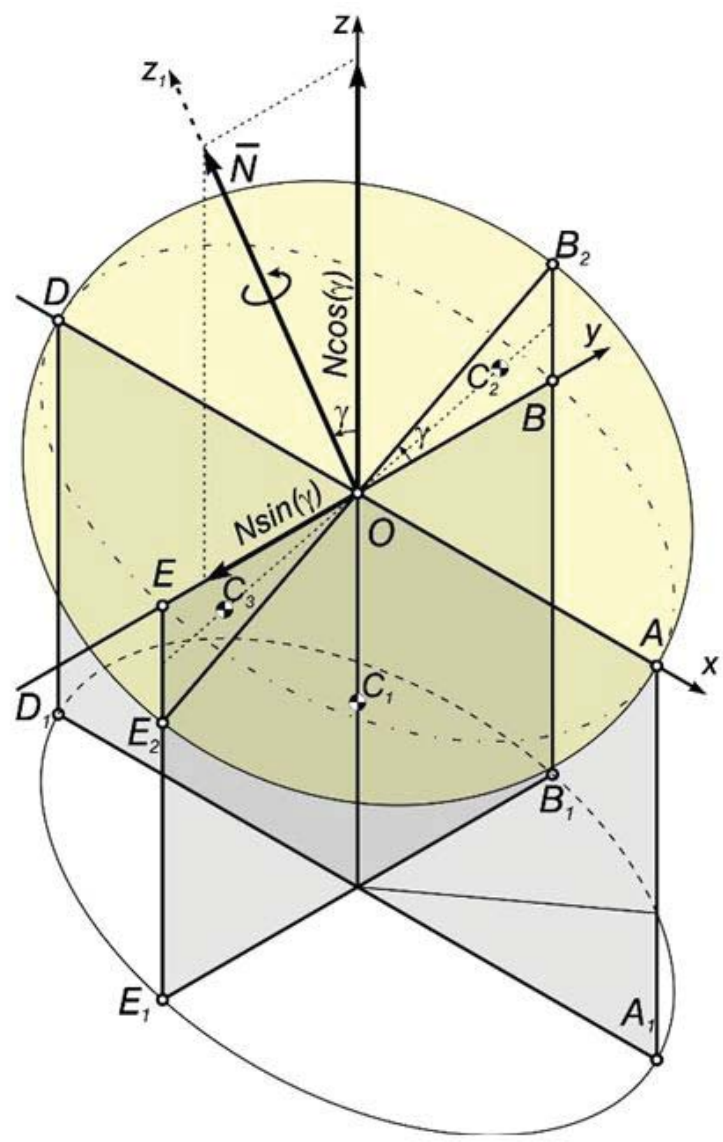

Figure 4: Lower part of the rotary support

$A B D B_{2}$ figure bounded by two intersecting planes and a lateral cylindrical surface has the coordinates of center of gravity $C_{2}$ and the volume equal to $[8,9]$

$\left.x_{2}=0 ; y_{2}=3 \pi R / 16 ; z_{2}=3 \pi R \cdot \operatorname{tg} \gamma\right) / 32$;

$V_{2}=2 R^{3} \cdot \operatorname{tg} \gamma / 3$

A similar figure $A D E_{2} A E D$ has the coordinates of center of gravity $C_{3}$ and the volume equal respectively to

$\left.x_{3}=0 ; y_{3}=-3 \pi R / 16 ; z_{3}=-3 \pi R \cdot \operatorname{tg} \gamma\right) / 32$

$V_{2}=-2 R^{3} \cdot \operatorname{tg} \gamma / 3$

where the volume of the 'cut out' part is negative.

The coordinates of center of gravity $\mathrm{C}_{H}$ of the lower part $A B_{2} D E_{2} A_{1} B_{1} D_{1} E_{1}$ of the rotary support, found as a weighted average of three parts, are

$x_{H}=0 ; y_{H}=\frac{1}{4} R \frac{B B_{2}}{B B_{1}}$

$z_{H}=B B_{1}\left[-\frac{1}{2}+\frac{1}{2}\left(\frac{B B_{2}}{B B_{1}}\right)^{2}\right]=-\frac{h_{1}}{2}+\frac{1}{8} \cdot \frac{R^{2}}{h_{1}} \cdot \operatorname{tg}^{2} \gamma$
In the initial position, center of gravity $C_{B}$ of upper part $\mathrm{B}_{2}{ }_{2} \mathrm{KNE}_{2}{ }_{2}$ is located symmetrically to point $\mathrm{CH}$ relative to the origin of coordinates $\mathrm{O}$ (Fig. 3). After turning this upper part by $180^{\circ}$ around axis $O Z_{1}$, its center of gravity $C B$ moves symmetrically with axis $O Z_{1}$ to position $C^{\prime} B$.

In this case, center of gravity of the load $C_{w}$ moves to position $\mathrm{C}^{\prime}{ }_{w}$. Therefore, the magnitude of the moment of stability is changed by $P \cdot O C_{W}^{\prime} \cdot \sin (2 \gamma)$. Thus

$P \cdot O C_{W}^{\prime} \cdot \sin (2 \gamma)=P\left[\frac{B_{1} K}{2}+\frac{L S}{2}\right] \cdot \sin (2 \gamma)$

where $O$ is the point of intersection of the axes of coordinates through which axis $O Z_{1}$ passes, against which the rotation of part $B_{2}{ }_{2} K N E_{2}^{\prime}$ is performed.

Consequently, the use of a rotary support in the exosystem can lead to a change in the moment of stability by the magnitude proportional to the product of the sine of the double angle $y$ and the sum of the rotary support device half-thickness and the load layer half-thickness.

\section{CONCLUSIONS}

Based on the foregoing, and also taking into account the articles [10,11, 12], the following conclusions can be made:

1. With a sharp change in the direction of movement (for small radii of curvature of the trajectory) the condition of lateral stability will be violated, the exoskeleton will lose its lateral stability and will turn over.

2. To increase the moment of stability, it is advisable to tilt the load perpendicular to the trajectory of movement in the direction of its rotation, using a rotary support.

3. In order to increase the maximum permissible speed of movement by $10 \%$, preventing the exoskeleton from tilting on its side (while maintaining the height of center of gravity $\mathrm{H}$ of the entire system and radius $R$ of the curvature of the trajectory), width $L$ of the base platform must be increased by $21 \%$.

4. The speed of movement of the exoskeleton should not exceed the maximum allowable speed, determined against the condition of lateral stability.

\section{ACKNOWLEDGEMENT}

The work was performed as part of the implementation of a comprehensive project to create high-tech production "Creating high-tech production of multifunctional robotic exoskeleton for medical purposes ("REM")", code 2017218-09-1807, approved by the government decree of the Russian Federation No. 218 of 9 April 2010. 


\section{REFERENCES}

1. Kapustin, A.V., Loskutov, Yu.V., Kudryavtsev, I.A., \& Belogusev, V.N., (2018). Methods to realize stable walking of rehabilitation exoskeleton. Vestnik of the Volga State University of Technology. Ser.: Materials. Constructions. Technologies, 3, 44-54.

2. Formal'sky, A.M., (2014). Motion control of unstable objects. Moscow: PHYSMATLIT.

3. Zhang, T., Tran, M., \& Huang, H. (2018). Design and Experimental Verification of Hip Exoskeleton with Balance Capacities for Walking Assistance. IEEE/ ASME Transactions on Mechatronics, 23(1), 274285. doi:10.1109/TMECH.2018.2790358

4. Martínez, A., Lawson, B., \& Goldfarb, M. (2018). A Controller for Guiding Leg Movement During Overground Walking With a Lower Limb Exoskeleton. IEEE Transactions on Robotics, 34(1), 183-193. doi:10.1109/TRO.2017.2768035

5. Andrianov, Yu.S., Kapustin, A.V., Egorov, A.V., \& Fishchenko, P.A., (2018). The effect of a sequence of conditions on the transformation of the state of the system. Innovations in life, 2, 69-83.

6. Kapustin, A.V., Loskutov, Yu.V., Skvortsov, D.V., Nasybullin, A.R., Klyuzhev, K.S., \& Kudryavtsev, A.I., (2018). Circuitry of the system for controlling a rehabilitation exoskeleton for medicinal purposes. Vestnik of the Volga State University of Technology. Ser.: Radio Engineering and Infocommunication Systems, 2, 77-86.
7. Loskutov, Y.V., Kapustin, A.V., Klyuzhev, K.S., Kudryavtsev, A.I., Loskutov, M.Y., \& Fadeev, A.M. (2017). Computer simulation of regular walking based on the kinematic analysis of movements and the synthesis of exoskeleton control algorithms. Vestnik of the Volga State University of Technology. Ser.: Radio Engineering and Infocommunication Systems, 3, 47-60.

8. Andrianov, D.Yu., \& Fishchenko, P.A., (2018). Calculation of the volume of the body by the method of splitting into elementary pyramids. U: Creativity of the young to scientific progress, 2018, Yoshkar-Ola. VSUT.53-55.

9. Andrianov, D.Yu., Kudryavtsev, A.I., \& Fishchenko, P.A., (2018). Estimation of the coordinates of element barycenter of rotation gear. U: Proceedings of the Volga State University of Technology. Ser.: Technological, 2018. Yoshkar-Ola: VSUT.52-56.

10. Jatsun, S.F., Savin, S.I., Jatsun, A.S., \& Malchikov, A.V., (2016). Study of controlled frontal plane motion of an exoskeleton in the vertical balance recovery regime. Extreme robotics, 1, 236-245.

11. Barbareschi, G., Richards, R., Thornton, M., Carlson, T., \& Holloway, C. (2015). Statically vs dynamically balanced gait: Analysis of a robotic exoskeleton compared with a human. U: 37th Annual International Conference of the IEEE Engineering in Medicine and Biology Society (EMBC). 6728-6731. doi:10.1109/EMBC.2015.7319937

12. Hof A.L., Gazendam M.G.J., \& Sinke W.E., (2005). The condition for dynamic stability. Journal of Biomechanics, 38(1), 1-8. 